\title{
THE IMPROVED TS-BASE APPROACHES WITH INTERFERENCE COMPENSATION AND THEIR EVALUATIONS FOR SPEECH ENHANCEMENT
}

\author{
Junfeng Li $^{1}$, Shuichi Sakamoto ${ }^{2}$, Satoshi Hongo ${ }^{3}$, Masato Akagi ${ }^{1}$ and Yôiti Suzuki ${ }^{2}$ \\ ${ }^{1}$ School of Information Science, Japan Advanced Institute of Science and Technology \\ ${ }^{2}$ Research Institute of Electrical Communication, Tohoku University \\ ${ }^{3}$ Department of Design and Computer Application, Miyagi Natational Collage of Technology
}

\begin{abstract}
We previously proposed a Two-Stage BinAural Speech Enhancement with Wiener Filter (TS-BASE/WF) approach for hearing aids in adverse environments [6]. In TS-BASE/WF, the interfering signal is estimated by cancelling the target signal through an adaptive filter in the first stage and a timevariant Wiener filter is applied to enhance the target signal in the second stage. In this paper, we introduce an interference compensation approach, which is applied to the adaptive-filter output, to further improve the estimation accuracy of the interfering signal. The performance of TS-BASE with different speech enhancers is then investigated in different conditions. Experimental results show that the improved TS-BASE algorithms with interference compensation outperform the original TS-BASE algorithms, and that TS-BASE/WF gives the higher speech enhancement performance over the improved TS-BASE algorithms with other speech enhancers.
\end{abstract}

Index Terms - TS-BASE, Adaptive filter, Interference compensation, Speech Enhancer.

\section{INTRODUCTION}

Though persons with normal hearing can understand speech in severely noisy environments, hearing-impaired persons have great difficulty in understanding speech in such conditions due to the hearing loss and the annoying acoustic noise. To facilitate the hearing of hearing-impaired persons, hearing aids have been designed and widely used. One of the main problems for hearing-aid users is the reduction of speech intelligibility in real-life noisy environments. To deal with this problem, efficient speech enhancement techniques have to be integrated into hearing-aid signal processing [1].

A large number of speech enhancement algorithms have so far been reported for hearing aids [1]. The generalized sidelobe canceller (GSC) was extended to binaural scenarios for hearing aids [2]. Campbell et al. applied a sub-band GSC beamformer to binaural noise reduction for hearing aids [3].

This study is supported by a Grant-in-Aid for Young Scientists (B) (No. 19700156) from the Ministry of Education, Science, Sports and Culture of Japan.
Moreover, Suzuki et al. suggested to introduce binaural cues into the constraints of an adaptive beamformer [4]. The major problem associated with these algorithms is the low noise reduction performance in multiple-noise-source environments. In recent years, Nakashima et al. presented a frequency domain binaural model based on interaural phase and level differences [5]. More recently, we proposed a Two-Stage BinAural Speech Enhancement with Wiener Filter (TS-BASE/WF) approach that combines interfering signal estimation by cancelling the target signal through adaptive filtering and a consequent stage that controls the transfer function of a time-variant Wiener filter [6].

In the previous TS-BASE/WF approach, the targetcancelled signal at the adaptive-filter output was simply regarded as the estimate of the interfering signal component in the input mixture signal. However, this is not the case due to the effect of the adaptive filters on the interference component which results in the difference of the interference component in the adaptive-filter output from that in the input mixture. In this paper, we introduce an interference compensation approach to compensate this difference by minimizing the difference in MMSE sense, and is further integrated into our previous TS-BASE approach. The concentration is then paid to the experimental investigations of the TS-BASE with different speech enhancement approaches with/without interference compensation. Experimental results confirm the effectiveness of the interference compensation in improving the speech enhancement performance of the TS-BASE algorithms and the higher performance of TS-BASE/WF with interference compensation.

\section{SIGNAL MODEL}

For hearing aids in real environments, the microphone signals at two ears, $X_{i}(k, \ell)(i=L, R)$, are corrupted by the uncorrelated interfering signals, $N_{i}(k, \ell)$, given by

$$
X_{i}(k, \ell)=S_{i}(k, \ell)+N_{i}(k, \ell),
$$

where $k$ and $\ell$ denote the frequency bin index and the frame index, respectively; $S_{i}(k, \ell)=H_{i}(k) S(k, \ell)$ is the corre- 


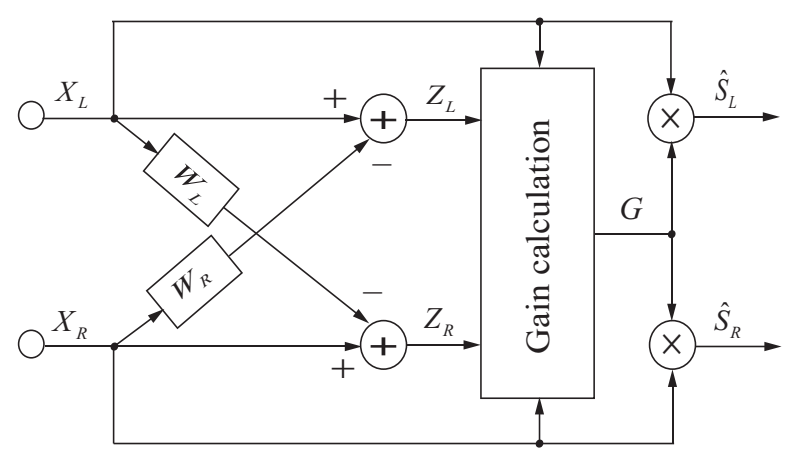

Fig. 1. Block diagram of the TS-BASE approach.

sponding short-time Fourier transforms (STFTs) of the observed target signal. $H_{i}(k)$ denotes the transfer functions between the target sound source to the head, known as headrelated transfer function (HRTF).

\section{ORIGINAL TS-BASE APPROACH}

\subsection{Estimation of interfering signal}

The objective of this part is to estimate interfering signal by cancelling the target signal, as shown in Fig. 1. In order to cancel the target signal and produce the interference-only output, we proposed to exploit two adaptive filters, $W_{L}$ and $W_{R}$, in the left and right channels. In the TS-BASE/WF approach, the adaptive filters are pre-learned using a white noise sequence of $10 \mathrm{~s}$ in the absence of interfering signal. The binaural input signals were generated by convolving the white noise sequence with the corresponding head-related impulse responses (HRIRs). With the generated binaural signals, two adaptive filters, $W_{L}(k, \ell)$ and $W_{R}(k, \ell)$, were obtained by using the normalized least mean square (NLMS) [6].

After determining the adaptive filters, their coefficients are fixed and applied to the observed mixture signals in the presence of interfering signal. As a result, the target-cancelled signals (i.e., the adaptive-filter outputs) are derived by subtracting the filter-calibrated inputs from the microphone signals, given by [6]

$$
\begin{aligned}
& Z_{L}(k, \ell) \approx N_{L}(k, \ell)-W_{R}(k, \ell) N_{R}(k, \ell), \\
& Z_{R}(k, \ell) \approx N_{R}(k, \ell)-W_{L}(k, \ell) N_{L}(k, \ell) .
\end{aligned}
$$

From Eqs. (2) and (3), we can observe that the target signal has been cancelled, producing the interference-only outputs.

\subsection{Enhancement of target signal}

For hearing-aid application, the system that outputs the binaural signals is much preferred. In the previously proposed TS-BASE approach, the target-cancelled signals, $Z_{i}(k, \ell)$, are used as interference estimate parameters to control the gain function of a speech enhancer shared in both channels. Three traditional speech enhancement approaches were utilized for enhancing the target signal, such as, Wiener filter, minimum mean sqaure error short-time spectral amplitude (MMSESTSA) [7], and minimum mean square error log-spectral amplitude (MMSE-LSA) [8]. Their gain functions are given by ( $k$ and $\ell$ are omitted for simplicity.)

$$
\begin{aligned}
G_{\mathrm{WF}} & =1-\frac{\xi}{1+\xi} \\
G_{\mathrm{STSA}} & =\Gamma(1.5) \frac{\sqrt{\nu}}{\gamma} \exp \left(-\frac{\nu}{2}\right)\left[(1+\nu) I_{0}\left(\frac{\nu}{2}\right)+\nu I_{1}\left(\frac{\nu}{2}\right)\right],
\end{aligned}
$$

$$
G_{\mathrm{LSA}}=\frac{\xi}{1+\xi} \exp \left(\frac{1}{2} \int_{v}^{\infty} \frac{e^{-t}}{t} d t\right)
$$

where $\Gamma(\cdot)$ represents the Gamma function (with $\Gamma(1.5)=$ $\sqrt{\pi} / 2), I_{0}(\cdot)$ and $I_{1}(\cdot)$ are the zeroth and first order modified Bessel functions, $\nu$ is defined as $\frac{\xi}{1+\xi} \gamma, \xi$ and $\gamma$ are the a priori SNR and the a posteriori SNR, defined as [7]

$$
\begin{aligned}
\xi & =\frac{E\left[S_{L} S_{L}^{*}+S_{R} S_{R}^{*}\right]}{E\left[Z_{L} Z_{L}^{*}+Z_{R} Z_{R}^{*}\right]}, \\
\gamma & =\frac{X_{L} X_{L}^{*}+X_{R} X_{R}^{*}}{E\left[Z_{L} Z_{L}^{*}+Z_{R} Z_{R}^{*}\right]},
\end{aligned}
$$

where the superscript ${ }^{*}$ is the conjugative operator, $E$ is the expected value operator. The a priori SNR $\xi$ is updated with the decision-directed mechanism [7]. With different speech enhancers given in Eqs.(4)-(6), the original corresponding TS-BASE approaches are hereafter named as TS-BASE/WF for using Wiener filter, TS-BASE/STSA for using MMSESTSA filter and TS-BASE/LSA for using MMSE-LSA filter.

\section{IMPROVED TS-BASE APPROACH}

\subsection{Compensation of interfering signal estimates}

As mentioned in Section 3, the function of the adaptive filter is to estimate the interfering signal by cancelling the target signal component from the observed mixture signal. In the original TS-BASE/WF approach, the target-cancelled signal at the adaptive filter output was supposed as the estimate of the interfering signal to calculate the gain function of Wiener filter [6]. Because of the filtering effect introduced by the adaptive filter, the target-cancelled signal must be different from the interference component in the observed signal, which results in the low interference estimation accuracy, and further the low reduction or high speech distortion.

To deal with this problem, we introduce a compensation approach for the accurate interference estimation based on the target-cancelled signal. In the proposed interference compensation approach, a time-variant frequency-dependent factor, $C_{i}(k, \ell)$, is exploited for mapping the target-cancelled signals 
(i.e., the adaptive-filter outputs) to the noise components in the input mixture signals. The compensation factor $C_{i}(k, \ell)$ is derived by the minimizing the mean square error between the target-cancelled signal and the input mixture signal. The cost function for optimal compensation factor is given by

$C_{i}(k, \ell)=\arg \min E\left[X_{i}(k, \ell)-N_{i}(k, \ell) C_{i}(k, \ell)\right] \cdot i=L, R$

The optimal compensation factor is found by setting the derivative of the cost function with respect to the factor $C_{i}(k, \ell)$ to zero, given by

$$
C_{i}^{\mathrm{opt}}(k, \ell)=\frac{\phi_{X_{i} Z_{i}}(k, \ell)}{\phi_{Z_{i} Z_{i}}(k, \ell)}, \quad i=L, R
$$

where $\phi_{X_{i} Z_{i}}(k, \ell)$ and $\phi_{Z_{i} Z_{i}}(k, \ell)$ are the cross-correlation and auto-correlation power spectral densities (PSD).

Note that since the target-cancelled signal and the interference component of input noisy signal are obtained by filtering the same source signal, the compensation factor $C_{i}(k, \ell)$ is dependent on the incidence directions of the target and interference signals. Therefore, $C_{i}(k, \ell)$ is more stationary parameters than the characteristics of the signal PSDs because the sound sources move continuously and slowly.

\subsection{Improved gain calculation of speech enhancers}

With the more accurate interference estimate processed by our compensation approach, the improved estimates of the $a$ priori SNR and the a posteriori SNR are re-calculated as

$$
\begin{aligned}
& \xi^{\mathrm{imp}}=\frac{E\left[S_{L} S_{L}^{*}+S_{R} S_{R}^{*}\right]}{E\left[\left(C_{L} Z_{L}\right)\left(C_{L} Z_{L}\right)^{*}+\left(C_{R} Z_{R}\right)\left(C_{R} Z_{R}\right)^{*}\right]}, \\
& \gamma^{\mathrm{imp}}=\frac{X_{L} X_{L}^{*}+X_{R} X_{R}^{*}}{E\left[\left(C_{L} Z_{L}\right)\left(C_{L} Z_{L}\right)^{*}+\left(C_{R} Z_{R}\right)\left(C_{R} Z_{R}\right)^{*}\right]}
\end{aligned}
$$

Substituting Eqs.(11) and (12) into Eqs.(4)-(6), the gain function of the improved traditional speech enhancement approaches, denoted as $G_{\mathrm{WF}}^{\text {imp }}, G_{\mathrm{STSA}}^{\text {imp }}$ and $G_{\mathrm{LSA}}^{\text {imp }}$, can be easily calculated. The corresponding TS-BASE approaches are named as TS-BASE/WF imp $_{\text {, TS-BASE/STSA }}$ imp and TSBASE/LSA $A_{\text {imp }}$, respectively.

\section{EXPERIMENTS AND RESULTS}

In this section, we examine the performance of the TS-BASE approaches with/without interference compensation using the different speech enhancers including TS-BASE/WF,

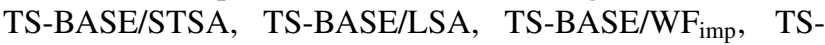
BASE/STSA $A_{i m p}$ and TS-BASE/LSA $A_{i m p}$, in the one- and multiple-noise-source environments. The studied binaural speech enhancement algorithms was evaluated in terms of the objective quality measures: segmental SNR (SEGSNR) and log-spectral distance (LSD) [9].

\subsection{Experimental configuration}

To evaluate the effectiveness of the studied algorithms, two noise acoustic environments, one-noise-source and threenoise-source conditions, were generated. In both environments, ten Japanese sentences were used as target signals and other thirty sentences as interfering signals. In our experiments, the head-related impulse response (HRIRs) were obtained from MIT media lab. [10]. The target sound source was placed in the front of the dummy head (i.e., $\mathrm{DOA}=0^{\circ}$ ), and the three interfering sources were located with DOAs of $-60^{\circ}, 60^{\circ}, 30^{\circ}$. The observed signals at two ears were created by convoluting the source (target and interference) signals with the corresponding HRIRs. In the one-noisesource condition, the noisy signals were obtained by summing the observed interfering signals with DOA of $60^{\circ}$ and the target signals at different global SNRs $[0,15] \mathrm{dB}$ with the step of $5 \mathrm{~dB}$. In the three-noise-source conditions, the interfering signals at two ears were first generated by mixing the individually observed three interfering signals, and then added to the target signals at the global SNRs same as in the one-noise-source condition.

\subsection{Experimental results}

The experimental results of SEGSNR averaged across all tested sentences in the one- and three-noise-source conditions are given in Table 1. The SEGSNR results in Table 1 demonstrate that the studied TS-BASE algorithms even without interference compensation still result in higher SEGSNRs, corresponding to the higher speech quality of the enhanced signals, compared with the noisy input signals in all tested conditions. Among the TS-BASE algorithms without interference compensation, the TS-BASE/WF approach illustrates the highest SEGSNR improvements in all tested conditions. More importantly, by integrating the interference compensation, the performance of the TS-BASE algorithms is further improved. The improved TS-BASE/WF approach with interference compensation, named as TS-

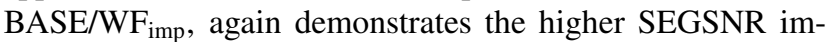
provements than the other improved TS-BASE algorithms using other speech enhancer in the second stage. On average, the TS-BASE/WF $\mathrm{Wmp}_{\text {imp }}$ approach yields the about about $0.7 \mathrm{~dB}$ and $0.4 \mathrm{~dB}$ SEGSNR improvements over the TS-

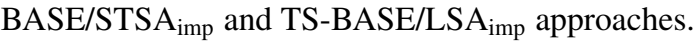

The experimental results of LSD averaged across all tested sentences in the one- and three-noise-source conditions are given in Table 2. The LSD results in Table 2 demonstrate that in all conditions the interference compensation provides the different degree of LSD decreases for all improved TSBASE algorithms regardless of the used speech enhancers in the second stage. Concerning the improved TS-BASE algorithms, the lowest LSDs are achieved by TS-BASE/WF imp $_{\text {in }}$ in the one-noise-source condition and by TS-BASE/LSA $\mathrm{imp}_{\mathrm{im}}$ in the three-noise-source condition, respectively. While, the 
Table 1. Segmental SNR $[\mathrm{dB}]$ of the noisy signal, the enhanced signals processed by TS-BASE/WF, TS-BASE/STSA, TS-BASE/LSA

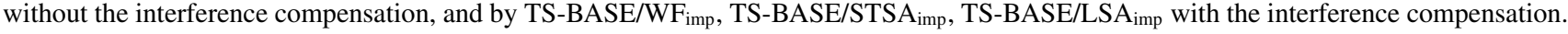

\begin{tabular}{|c|c|c|c|c|c|c|c|c|c|}
\hline \multirow[t]{2}{*}{ Algorithm } & \multicolumn{4}{|c|}{ One-Noise-Source Condition } & \multicolumn{4}{|c|}{ Three-Noise-Source Condition } & \multirow[t]{2}{*}{ Averag } \\
\hline & 0 & 5 & 10 & 15 & 0 & 5 & 10 & 15 & \\
\hline Noisy & -1.94 & -0.35 & 3.67 & 8.83 & -0.58 & 2.77 & 6.68 & 10.82 & 3.74 \\
\hline TS-BASE/WF & 4.19 & 6.39 & 9.14 & 12.04 & 2.88 & 5.21 & 7.93 & 10.86 & 7.33 \\
\hline TS-BASE/STSA & 2.76 & 5.48 & 8.40 & 11.31 & 2.43 & 4.96 & 7.69 & 10.64 & 6.71 \\
\hline TS-BASE/LSA & 3.07 & 5.67 & 8.51 & 11.39 & 2.63 & 5.01 & 7.67 & 10.58 & 6.82 \\
\hline TS-BASE/WF imp & 4.51 & 6.96 & 9.69 & 12.40 & 3.23 & 5.82 & 8.59 & 11.54 & 7.85 \\
\hline TS-BASE/STSA $_{\text {imp }}$ & 2.96 & 5.85 & 8.87 & 11.72 & 2.66 & 5.40 & 8.26 & 11.26 & 7.13 \\
\hline TS-BASE/LSA & 3.49 & 6.27 & 9.20 & 12.02 & 3.01 & 5.64 & 8.40 & 11.37 & 7.43 \\
\hline
\end{tabular}

Table 2. Log-spectral distance [dB] of the noisy signal, the enhanced signals processed by TS-BASE/WF, TS-BASE/STSA, TS-BASE/LSA without the interference compensation, and by TS-BASE/WF imp TS-BASE/STSA $_{\text {imp }}$, TS-BASE/LSA imp $_{\text {with }}$ the interference compensation.

\begin{tabular}{|c|c|c|c|c|c|c|c|c|c|}
\hline \multirow[t]{2}{*}{ Algorithm } & \multicolumn{4}{|c|}{ One-Noise-Source Condition } & \multicolumn{4}{|c|}{ Three-Noise-Source Condition } & \multirow[t]{2}{*}{ Average } \\
\hline & 0 & 5 & 10 & 15 & 0 & 5 & 10 & 15 & \\
\hline Noisy & 7.49 & 6.52 & 5.59 & 4.25 & 7.76 & 5.69 & 3.95 & 2.60 & 5.48 \\
\hline TS-BASE/WF & 4.92 & 4.05 & 3.25 & 2.48 & 4.91 & 3.84 & 2.88 & 2.06 & 3.55 \\
\hline TS-BASE/STSA & 4.89 & 3.96 & 3.17 & 2.43 & 4.67 & 3.61 & 2.69 & 1.92 & 3.42 \\
\hline TS-BASE/LSA & 4.73 & 3.88 & 3.13 & 2.41 & 4.64 & 3.62 & 2.72 & 1.96 & 3.39 \\
\hline TS-BASE/WF imp & 4.38 & 3.59 & 2.84 & 2.15 & 4.37 & 3.67 & 2.49 & 1.76 & 3.16 \\
\hline TS-BASE/STSA $_{\mathrm{imp}}$ & 4.92 & 3.93 & 3.07 & 2.31 & 4.56 & 3.45 & 2.52 & 1.77 & 3.32 \\
\hline TS-BASE/LSA ${ }_{\text {imp }}$ & 4.53 & 3.66 & 2.89 & 2.19 & 4.34 & 3.33 & 2.45 & 1.74 & 3.14 \\
\hline
\end{tabular}

average LSD result difference between TS-BASE/WF $\mathrm{Wmp}_{\text {imp }}$ and TS-BASE/LSA $\mathrm{im}_{\mathrm{im}}$ in the tested conditions is just $0.02 \mathrm{~dB}$.

On the whole, the interference compensation is effective in improving the performance of the original TS-BASE approaches in the sense of the increased SEGSNRs and the decreased LSDs in all tested conditions. Moreover, the im-

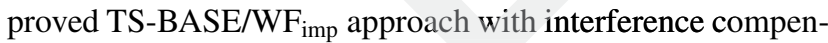
sation yields the higher speech enhancement performance.

\subsection{Discussions on sound localization ability}

In [6], it has been proved that the original TS-BASE algorithm is effective in preserving the ability of target signal source localization. Note that the improved TS-BASE algorithms with interference compensation have the same structure as the original TS-BASE algorithms. Therefore, it is believed that the improved TS-BASE algorithms are still able to localize the target sound sources.

\section{CONCLUSION}

In this paper, we further improved our previously proposed TS-BASE approach by integrating interference compensation, and investigated the different single-channel speech enhancement methods used as the enhancer for target signal. Experimental results show that the interference compensation improve the speech enhancement ability of the TS-BASE algorithms, and the improved TS-BASE/WF $\mathrm{Wmp}_{\text {imp }}$ demonstrates the higher performance in enhancing the target speech signal.

\section{REFERENCES}

[1] V. Hamacher, et al., "Signal processing in high-end hearing aids: State of the art, Challenges, and Future Trends," EURASIP Journal on ASP, vol. 18, pp. $2915-2929,2005$

[2] J.G. Desloge, et al., "Microphone-array hearing aids with binaural output-part I: Fixed-processing systems," IEEE Trans. SAP, vol. 5, pp. 529-542, 1997.

[3] D. Campbell and P. Shields, "Speech enhancement using sub-band adaptive Griffiths-Jim signel processing," Speech Comm., vol. 39, pp. 97-110, 2003.

[4] Y. Suzuki, et al., "New design method of a binaural microphone array using multiple constraints," IEICE Trans. Fundamentals, vol. E82-A, pp. 588-595, 1999.

[5] H. Nakashima, et al., "Frequency domain binaural model based on interaura phase and level differences," Acoustical Science and Technology, vol. 24, no. 4 , pp. 172-178, 2003.

[6] J. Li, et al., "A two-stage binaural speech enhancement approach for hearing aids with preserving binaural benefits in noisy environments," in Proc. Forum Acousticum 2008, Paris, France, June, 2008.

[7] Y. Ephraim, D. Malah,"Speech enhancement using a minimum mean-square error short-time spectral amplitude estimator," IEEE Trans. ASSP, vol. 32, pp. $1109-1121,1984$

[8] Y. Ephraim, D. Malah, "Speech enhancement using a minimum mean-square error log-spectral amplitude estimator," IEEE Trans. ASSP, vol. 33, pp. 443-445, 1985

[9] J. Li, et al., "Adaptive $\beta$-order generalized spectral subtraction for speech en hancement," Signal Processing, vol. 88, no. 11, pp. 2764-2776, 2008.

[10] http://sound.media.mit.edu/KEMAR.html. 